\title{
Tratamiento de la retrusión del tercio medio facial con cirugía ortognática combinada
}

\author{
Treatment of the middle third facial retrusion \\ with combined orthognathic surgery \\ Dr. Jorge David Serrano-Andrade,* Dr. Jacobo Felemovicius-Hermangus, * \\ CD. María Eliza González-Páder, ${ }^{\S}$ Dr. Francisco Javier Ramírez-Fernández
}

Palabras clave:

Cirugía ortognática

combinada, LeFort I, osteotomía mandibular

sagital, Angle III.

Keywords:

Combined orthognathic

surgery, LeFort I,

sagittal mandibular

osteotomy, Angle III.

${ }^{*}$ Residente de

Alta Especialidad

en procedimientos

avanzados en cirugía

ortognática. Servicio de

Cirugía Plástica.

\$ Médico adscrito del

Servicio de Cirugía

Plástica.

$\S$ Residente de tercer

año del Servicio de

Odontología.

" Médico pasante en

Servicio Social.

Hospital General «Dr.

Manuel Gea González».

Recibido:

18 noviembre 2020

Aceptado:

\section{RESUMEN}

La discrepancia anteroposterior entre el maxilar y la mandíbula, en la que la mandíbula ocluye por delante del maxilar, puede deberse a un defecto del hueso maxilar, a un exceso de la mandíbula o a una combinación de ambos. La maloclusión clase III, a pesar de tener una fuerte base genética, presenta etiología multifactorial, con prevalencia del $1-5 \%$ en la población blanca y cercana al $13 \%$ en asiáticos, considerada por la Organización Mundial de la Salud como el tercer lugar en problemas de salud oral. Presentamos el caso de un hombre de 23 años, con diagnóstico de maloclusión clase III de Angle con Retrusión del tercio medio facial. Se realiza cirugía ortognática combinada que comprendió osteotomía LeFort I de avance más osteotomía sagital para retroposición mandibular, aunado al tratamiento ortodóncico pre- $\mathrm{y}$ postoperatorio con fin de establecer estabilidad oclusal previo a la cirugía ortognática. Se consigue un cambio radical clínica y cefalométricamente del paciente, evidenciando un ángulo frontonasal, nasolabial y mentolabial esperados. Podemos concluir que la cirugía ortognática combinada representa un poderoso arsenal a nuestro alcance para poder tratar todas las alteraciones que afectan la oclusión dentaria, la armonía esquelética facial y la estética, que no logran ser tratadas con ortodoncia o un procedimiento ortognático simple.

\section{ABSTRACT}

Anteroposterior discrepancy between the maxilla and mandible, in which the mandible occludes in front of the maxilla, may be due to a maxillary bone defect, an excess of the mandible, or a combination of both. Class III malocclusion, despite having a strong genetic basis, presents multifactorial etiology, with a prevalence of $1-5 \%$ in the white population and close to $13 \%$ in Asians, considered by the World Health Organization as the third place in oral health problems. We present the case of a 23-year-old male patient with a diagnosis of Angle's class III malocclusion with retrusion of the midface. Combined orthognathic surgery was performed, which included advance LeFort I osteotomy plus sagittal osteotomy for mandibular retroposition, together with pre- and postoperative orthodontic treatment in order to establish occlusal stability prior to orthognathic surgery. A radical change is achieved clinically and cephalometrically in the patient, showing an expected frontonasal, nasolabial and mentolabial angle. We can conclude that combined orthognathic surgery represents a powerful arsenal at our disposal to be able to treat all the alterations that affect dental occlusion, facial skeletal harmony and aesthetics that cannot be treated with orthodontics or a simple orthognathic procedure.

\section{INTRODUCCIÓN}

$\mathrm{E}^{\mathrm{x}}$ xisten problemas faciales y dento-oclusales que resultan de la discrepancia en las relaciones entre el hueso maxilar y la mandíbula que conlleva a un desequilibrio de la cara, tanto estético como funcional.

Edward H. Angle en 1899 hipotetizó que la oclusión se basa en la relación entre los primeros molares permanentes del maxilar y de la

Citar como: Serrano-Andrade JD, Felemovicius-Hermangus J, González-Páder ME, Ramírez-Fernández FJ. Tratamiento de la retrusión del tercio medio facial con cirugía ortognática combinada. Cir Plast. 2021; 31 (1): 18-24. https://dx.doi.org/10.35366/101082 
mandíbula, determinando así que la oclusión normal es cuando la cúspide mesiobucal del primer molar maxilar ocluye en el surco bucal del primer molar mandibular.

Se describen tres clases de maloclusión, ${ }^{1}$ donde la clase III está definida cuando la cúspide mesiovestibular del primer molar maxilar se articula de forma distal al surco mesiovestibular del primer molar mandibular. El molar mandibular está posicionado mesial con relación al molar maxilar, ${ }^{1}$ caracterizándose por mostrar una retrusión del tercio medio facial, labio superior plano o con hundimiento y un ángulo nasolabial horizontalizado, incisivos laterales pequeños o ausentes y mordida cruzada en algunas ocasiones. En la cefalometría, el ángulo SNA está disminuido, el ángulo SNB aumentado o normal y el ángulo ANB siempre será negativo. ${ }^{2,3}$ En estos casos, la cirugía ortognática combinada con la osteotomía LeFort I puede restaurar estética y funcionalmente el esqueleto facial. ${ }^{4}$

La maloclusión clase III, a pesar de tener una fuerte base genética, presenta una etiología multifactorial, con una prevalencia del $1-5 \%$ en la población blanca y cercana al 13\% en poblaciones asiáticas. En Latinoamérica

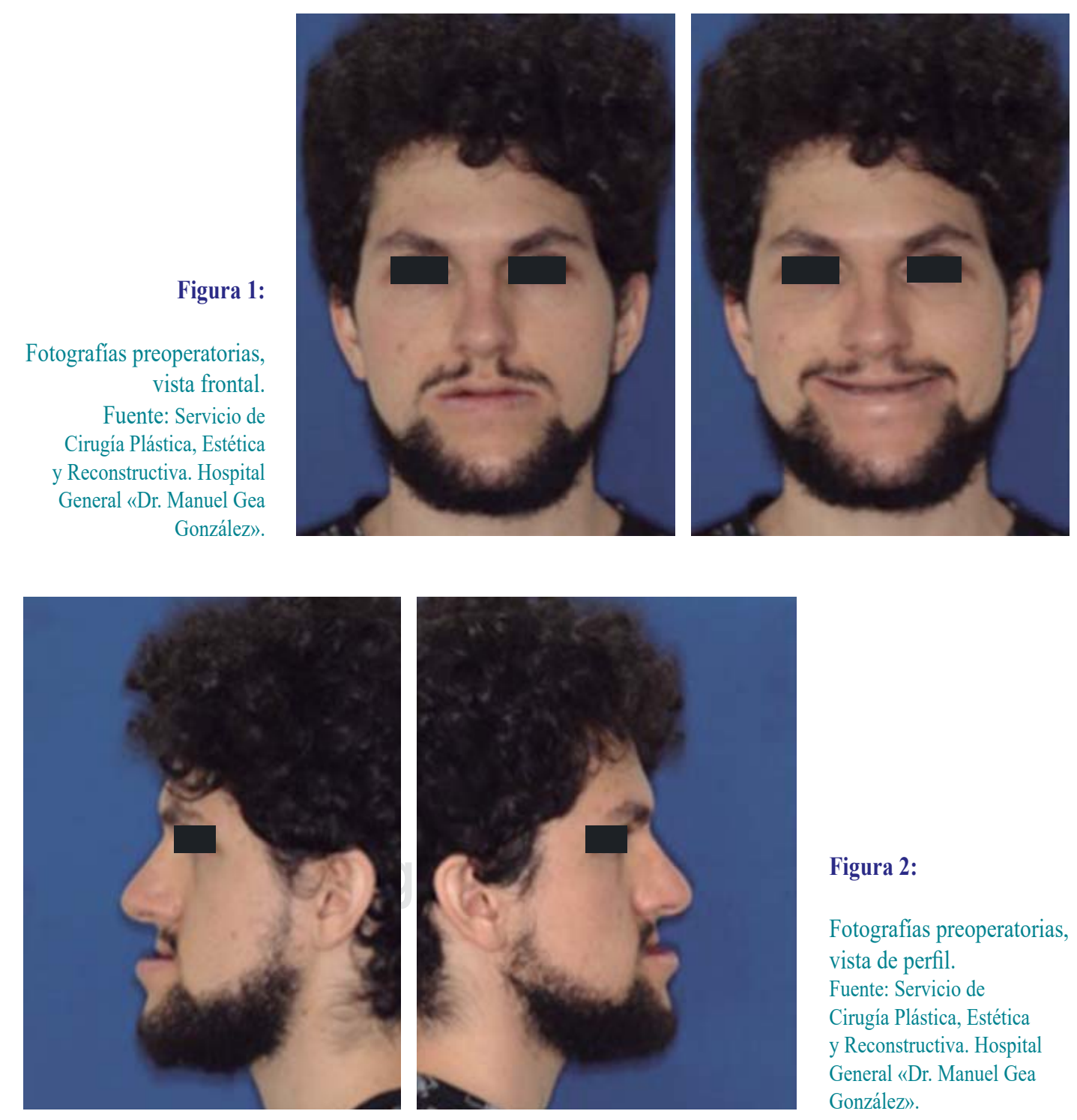



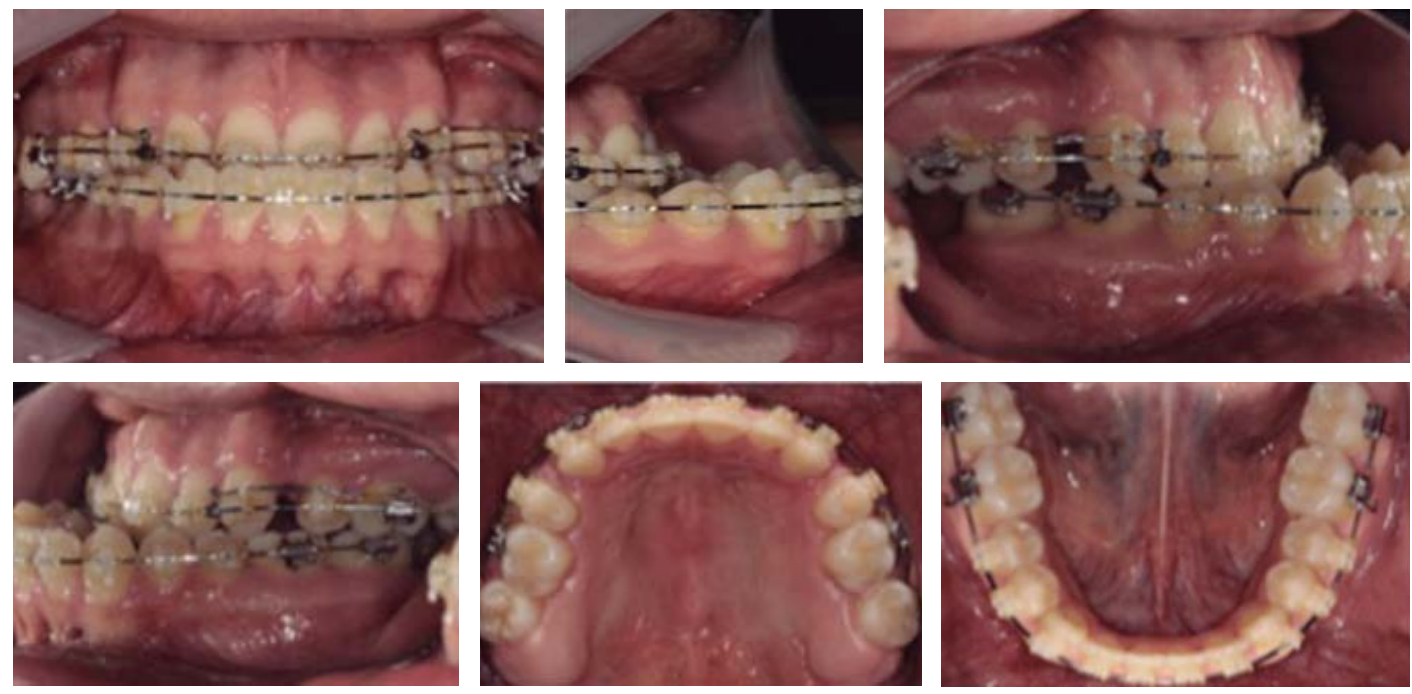

Figura 3: Mordida preoperatoria.

Fuente: Servicio de Ortodoncia. Hospital General «Dr. Manuel Gea González».

ocupan el tercer lugar en cuanto a prevalencia. ${ }^{5}$ Según la Organización Mundial de la Salud, ocupan el tercer lugar como problemas de salud oral, su prevalencia e incidencia son consideradas como problemas de salud pública. ${ }^{6}$

\section{CASO CLÍNICO}

Hombre de 23 años de edad, sin antecedentes patológicos de importancia, que ulterior a la exploración física en cara se evidencia implantación de cabello adecuada, con proyección malar disminuida y labios delgados, facies con ligera asimetría facial, perfil cóncavo, forma de cráneo normocéfalo y forma de cara mesoprosopa, mentón protruido, ángulo frontonasal $130^{\circ}$, ángulo nasolabial $127^{\circ}$ y ángulo mentolabial $88^{\circ}$ (Figuras 1 y 2 ).

En la cavidad oral se identifica un maxilar sobreexpandido dentoalveolarmente, extracción de primeros premolares superiores y desviación de la línea media superior 2.5 $\mathrm{mm}$ hacia la derecha. Maloclusión molar y canina clase III de Angle. Mordida cruzada anterior y overjet negativo de $21 \mathrm{~mm}$ (Figura 3).

En la cefalometría de Steiner se identifica ángulo SNA de $83^{\circ}$, ángulo SNB de $90^{\circ}$ y ángulo ANB de $8^{\circ}$ (Figura 4).
Se estableció el diagnóstico de maloclusión clase III de Angle más retrusión del tercio medio facial.

\section{Procedimientos realizados}

Se realiza la programación preoperatoria de cirugía ortognática combinada y se inicia con el tratamiento ortodóncico preoperatorio, que consistió en la colocación de dos tornillos en paladar para corregir la sobreexpansión dentoalveolar del maxilar superior, palatinizando los primeros y segundos molares superiores. Para buscar una estabilidad oclusal, se colocaron arcos $0.017 \times 0.025$ de acero, buscando la descompensación prequirúrgica. En seis meses se consiguió la estabilidad oclusal y se sometió a cirugía ortognática combinada: osteotomía Lefort I de avance maxilar, de $9 \mathrm{~mm}$, con rotación hacia la izquierda $3 \mathrm{~mm}$; osteotomía sagital mandibular de retroceso de $9 \mathrm{~mm}$, la que se llevó a cabo mediante:

Osteotomía LeFort I de avance y rotación: paciente en decúbito dorsal, con anestesia general e intubación nasotraqueal. Se infiltra lidocaína con epinefrina en región maxilar, nasal, vestibular y en articulación temporomandibular. Se realiza incisión vestibular $5 \mathrm{~mm}$ por encima de la unión mucogingival de primer 
molar a primer molar, después se diseca maxilar liberando la fosa piriforme hasta agujero infraorbitario. Marcaje y osteotomía LeFort tipo I con sierra reciprocante de contrafuertes nasomaxilar, cigomático maxilar y con cincel de Lambotte del contrafuerte pterigomaxilar, separando el vómer del septum nasal para realizar después disyunción pterigomaxilar con pinzas de Rowe. Colocación de férulas dentarias. Se fijan segmentos maxilares con

\begin{tabular}{|c|c|c|c|c|}
\hline & Valor & Norma & Desv. Std & Desv. Norm. \\
\hline \multicolumn{5}{|l|}{ Relación craneofacial-estructura craneal } \\
\hline Largo craneal $(\mathrm{mm})$ & 69.7 & 61.9 & 2.5 & $3.1^{* * *}$ \\
\hline Altura facial posterior (Go-CF) (mn) & 83.2 & 61.0 & 3.30 & $6.7^{* * * * * *}$ \\
\hline Deflección craneal $\left({ }^{\circ}\right)$ & 27.2 & 29.6 & 3.0 & -0.8 \\
\hline Ubicación del porión (mm) & -36.5 & -37.0 & 2.2 & 0.2 \\
\hline Posición del ramus $\left(^{\circ}\right)$ & 90.8 & 77.5 & 3.0 & $4.4^{* * * *}$ \\
\hline \multicolumn{5}{|l|}{ Relación craneofacial-posición mx } \\
\hline Profundidad maxilar (FH-NA) $\left(^{\circ}\right)$ & 90.4 & 93.4 & 3.0 & $-1.0^{*}$ \\
\hline Altura maxilar $(\mathrm{N}-\mathrm{CF}-\mathrm{A})\left({ }^{\circ}\right)$ & 50.9 & 59.6 & 3.0 & $-2.9^{* *}$ \\
\hline Plano SN-Palatal $\left({ }^{\circ}\right)$ & 2.9 & 7.3 & 3.5 & $-1.3^{*}$ \\
\hline \multicolumn{5}{|l|}{ Relación craneofacial-posición md } \\
\hline Ángulo facial $(\mathrm{FH}-\mathrm{NPo})\left({ }^{\circ}\right)$ & 97.4 & 91.6 & 3.0 & $1.9^{*}$ \\
\hline Eje facial-Ricketts (NaBa-PtGn) $\left({ }^{\circ}\right)$ & 97.3 & 89.2 & 3.5 & $2.3^{* *}$ \\
\hline $\mathrm{FMA}(\mathrm{MP}-\mathrm{FH})\left({ }^{\circ}\right)$ & 26.0 & 22.9 & 4.5 & 0.7 \\
\hline Altura total de la cara $(\mathrm{NaBa}-\mathrm{PmXi})\left({ }^{\circ}\right)$ & 55.9 & 60.0 & 3.0 & $-1.4^{*}$ \\
\hline Conicidad facial $\left({ }^{\circ}\right)$ & 56.6 & 68.5 & 3.5 & $-3.4 * * *$ \\
\hline \multicolumn{5}{|l|}{ Relación maxilomandibular } \\
\hline Convexidad (A-NPo) (mm) & -7.6 & 2.8 & 2.0 & $-5.2 * * * * *$ \\
\hline Longitud del corpus (Go-Gn) (mm) & 101.5 & 79.0 & 4.4 & $5.1 * * * * *$ \\
\hline Arco mandibular $\left(^{\circ}\right)$ & 34.6 & 34.7 & 4.0 & 0.0 \\
\hline Altura de la cara inferior (ANS-Xi-Pm) $\left(^{\circ}\right)$ & 45.8 & 44.5 & 4.0 & 0.3 \\
\hline \multicolumn{5}{|l|}{ Relaciones dentales - dentición $\mathrm{mx}$} \\
\hline Protrusión del incisivo U (U1-APo) (mm) & -11.0 & 6.7 & 2.3 & $-7.7^{* * * * * * *}$ \\
\hline $\mathrm{U} 1-\mathrm{FH}\left({ }^{\circ}\right)$ & 103.8 & 111.0 & 6.0 & $-1.2 *$ \\
\hline Inclinación del incisivo $\mathrm{U}(\mathrm{U} 1-\mathrm{APo})\left({ }^{\circ}\right)$ & 0.4 & 28.0 & 4.0 & $-6.9 * * * * * *$ \\
\hline U6-PT vertical (mm) & 30.7 & 21.0 & 3.0 & $3.2 * * *$ \\
\hline \multicolumn{5}{|l|}{ Relaciones dentales - dentición md } \\
\hline Protuberancia L1 (L1-APo) (mm) & 9.6 & 2.0 & 2.3 & $3.3^{* * *}$ \\
\hline $\mathrm{L} 1$ a A-Po $\left({ }^{\circ}\right)$ & 37.0 & 27.7 & 4.0 & $2.3^{* *}$ \\
\hline Extrusión de los incisivos mandibulares (mm) & 1.9 & 2.4 & 2.0 & -0.2 \\
\hline Ángulo del eje de la bisagra & 84.2 & 90.0 & 4.0 & $-1.5^{*}$ \\
\hline \multicolumn{5}{|l|}{ Relaciones dentales - dentición $\mathrm{mx} / \mathrm{md}$} \\
\hline Ángulo interincisal (U1-L1) $\left({ }^{\circ}\right)$ & 142.5 & 124.0 & 6.0 & $3.1^{* * *}$ \\
\hline Relación molar (mm) & -11.0 & -1.6 & 1.0 & $-9.4 * * * * * * *$ \\
\hline Sobremordida (mm) & -20.9 & 3.4 & 2.5 & $-9.7^{* * * * * * *}$ \\
\hline Sobremordida (mm) & 3.9 & 2.8 & 2.0 & 0.5 \\
\hline Plano Occ a FH $\left({ }^{\circ}\right)$ & 11.8 & 7.5 & 5.0 & 0.9 \\
\hline \multicolumn{5}{|l|}{ Estética } \\
\hline Labio inferior al plano $\mathrm{E}(\mathrm{mm})$ & -1.3 & -2.0 & 2.0 & 0.4 \\
\hline
\end{tabular}

placas sistema 2.0 en contrafuertes cigomático maxilar y nasomaxilar. Cierre de la mucosa vestibular.

Osteotomía sagital mandibular de retroceso: incisión de 1-2 cm por encima del plano oclusal, continuando hacia el primer molar con disección subperióstica del segmento mandibular a nivel del espacio retromolar, facilitando la exposición con separador de Obwegeser de cara lateral de cuerpo, ángulo y rama ascendente. Osteotomía sagital lateral con sierra de corte lateral en la rama ascendente de la mandíbula por la línea oblicua, hasta $1 \mathrm{~cm}$ por encima de la espina de Spix, para dejar así el pedículo neurovascular en el segmento medial. Se completa la osteotomía y se separan los segmentos con cincel. Se repite el procedimiento en el lado contrario. Se colocan férulas dentarias para alinear los segmentos en la posición deseada. Se realiza fijación de los segmentos mandibulares con tornillos bicorticales con técnica percutánea. Se cierran heridas en mucosa, previa revisión de los segmentos óseos y la oclusión según la planificación previa.

En el postquirúrgico, el paciente evidencia el cambio (Figuras 5 y 6), con un ángulo frontonasal de $130^{\circ}$, nasolabial de $99^{\circ}$ y mentolabial

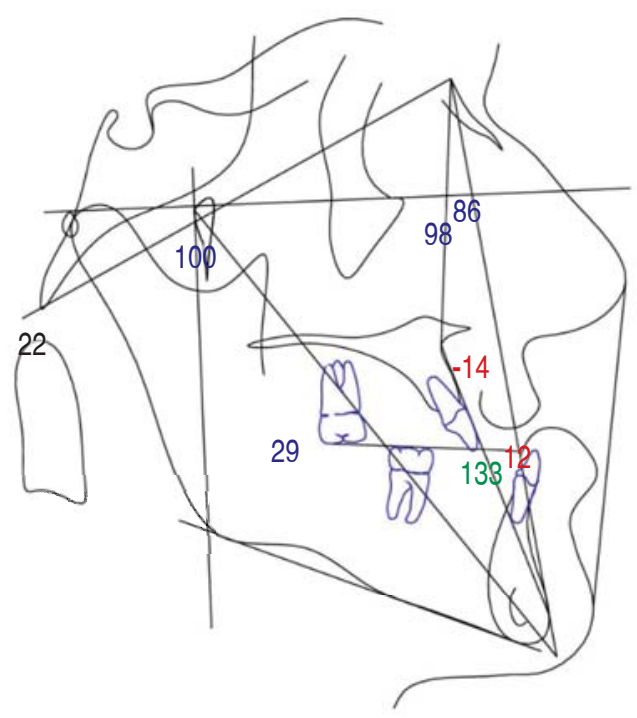

Figura 4: Cefalometría Ricketts preoperatoria.

Fuente: Servicio de Ortodoncia. Hospital General «Dr. Manuel Gea González». 

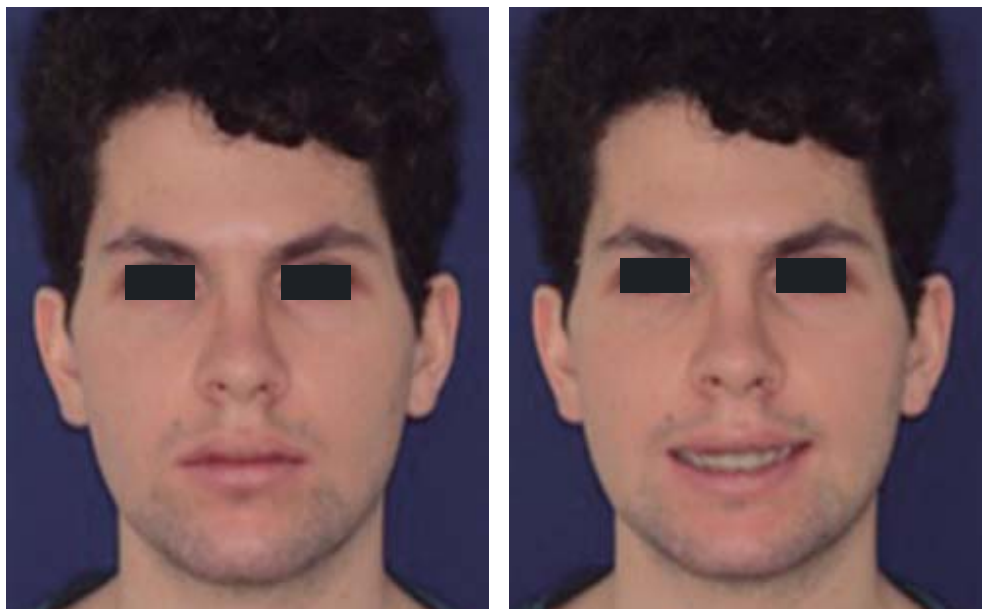

Figura 5: Fotografías postoperatorias, vista frontal.

Fuente: Servicio de Cirugía Plástica, Estética y Reconstructiva. Hospital General «Dr. Manuel Gea González».
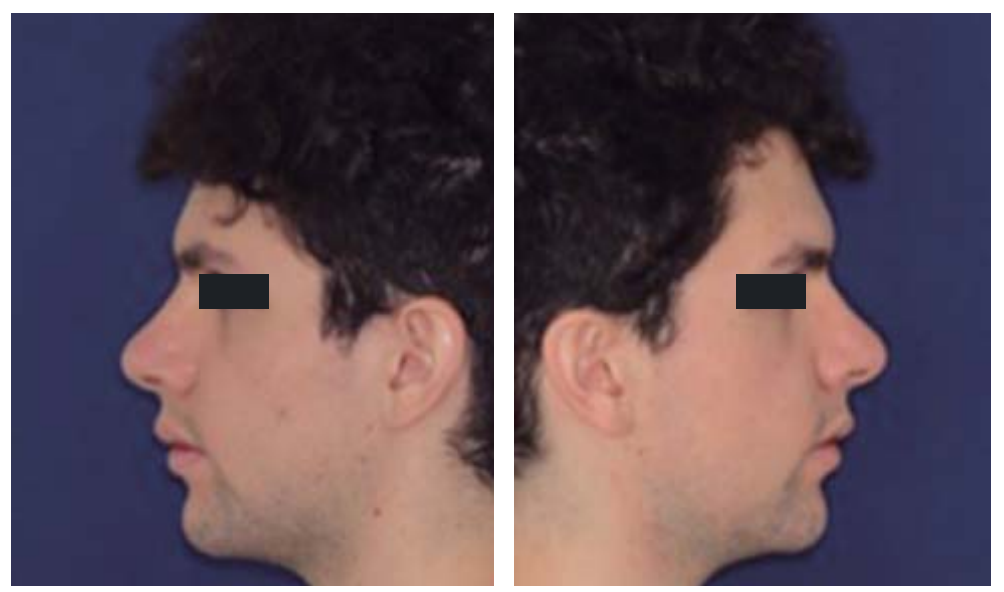

Figura 6: Fotografías postoperatorias, vista de perfil.

Fuente: Servicio de Cirugía Plástica, Estética y Reconstructiva. Hospital General «Dr. Manuel Gea González».
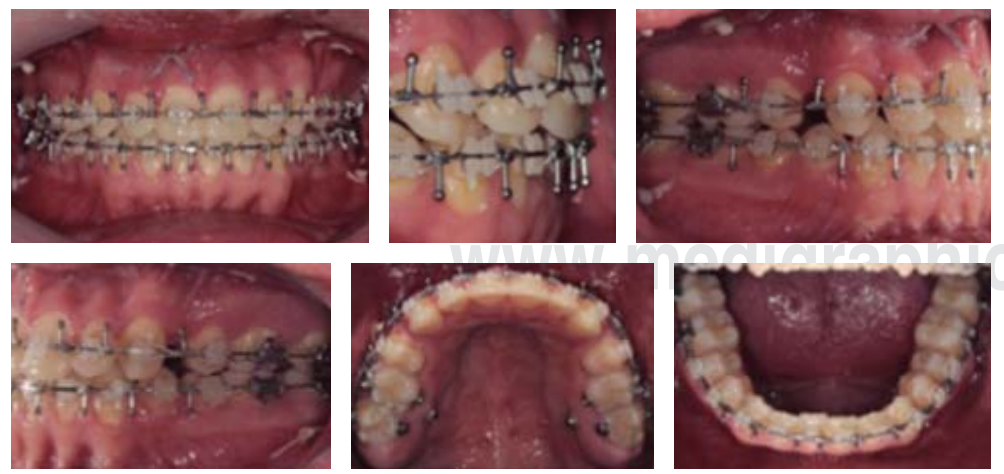

Figura 7: Mordida postoperatoria.

Fuente: Servicio de Ortodoncia. Hospital General «Dr. Manuel Gea González». de $120^{\circ}$, un overjet positivo de $2 \mathrm{~mm}$, oclusión clase I canina de Angle y clase II molar funcional de Angle.

En la actualidad, el paciente se encuentra en tratamiento ortodóncico postoperatorio en fase de cierre de espacios para después continuar con la fase de asentamiento y detallado (Figuras 7 a 14).

\section{DISCUSIÓN}

La exploración de cada uno de los componentes a tratar es indispensable para poder lograr un tratamiento adecuado, que incluye una evaluación funcional y estética. ${ }^{7,8}$

En la evaluación dental se deben identificar las discrepancias transversales y anteroposteriores. Para la evaluación del plano frontal y de perfil se debe dividir al paciente en tercios, con el objetivo de lograr el balance de todas estas regiones. ${ }^{3,7}$

El manejo multidisciplinario con el Servicio de Ortodoncia representa 18 a 24 meses de tratamiento quirúrgico, desde la fase de planeación inicial hasta la cirugía y colocación de retenedores, para mantener una estabilidad a largo plazo. ${ }^{9}$

Se han incluido los modelos con TAC3D que son programas de diseño asistidos por computadora (CAD) que ayudan a la preparación del paciente con un análisis cefalométrico, simulando los movimientos del maxilar o la mandíbula en cualquier dimensión, para la fabricación de las férulas quirúrgicas con esta misma tecnología, incluyendo las osteotomías y la fabricación de las guías de corte para mejorar la exactitud de las osteotomías. ${ }^{10}$ La planeación quirúrgica virtual ha demostrado tener una exactitud de $1 \mathrm{~mm}$, logrando ahorrar tiempo en comparación con la cirugía de modelos. También se ha demostrado acortamiento de las osteotomías y mejoría en la fijación.?

La apariencia de los tejidos blandos que cubren el esqueleto facial son el factor crucial que determina el resultado estético de la cirugía, los huesos deben ser posicionados de tal forma que brinden un óptimo soporte de los tejidos blandos. Así, la expansión esquelética anteroinferior reduce la laxitud de los tejidos blandos y suaviza los pliegues de la cara y la 

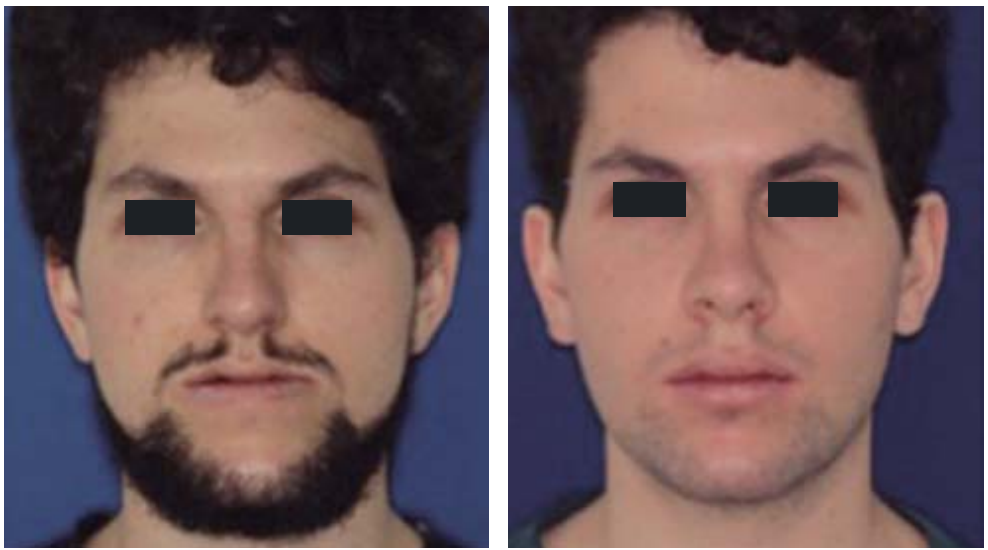

Figura 8: Fotografías comparativas pre- y postoperatorio, vista frontal. Fuente: Servicio de Cirugía Plástica, Estética y Reconstructiva. Hospital General «Dr. Manuel Gea González».
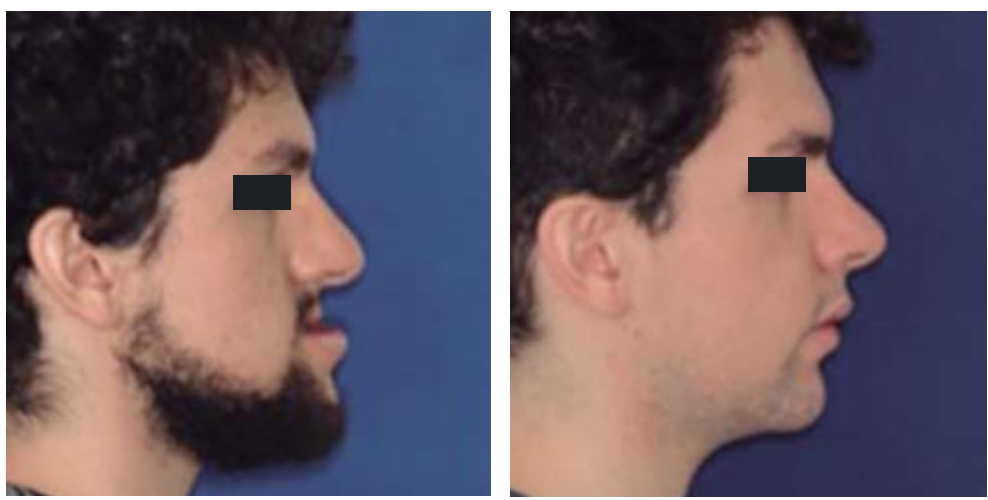

Figura 9: Fotografías comparativas pre- y postoperatorio, perfil derecho. Fuente: Servicio de Cirugía Plástica, Estética y Reconstructiva. Hospital General «Dr. Manuel Gea González»
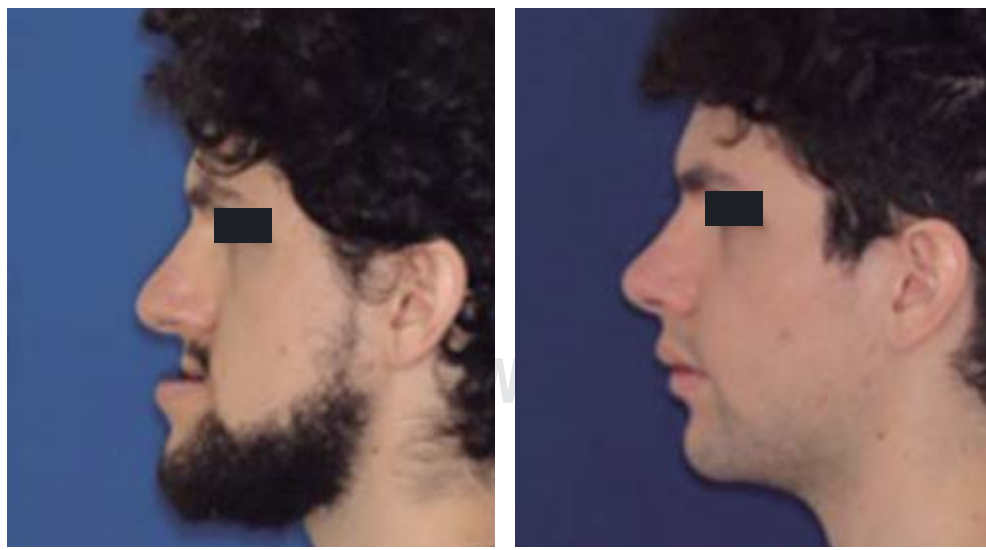

Figura 10: Fotografías comparativas pre- y postoperatorio, perfil izquierdo. Fuente: Servicio de Cirugía Plástica, Estética y Reconstructiva. Hospital General «Dr. Manuel Gea González».

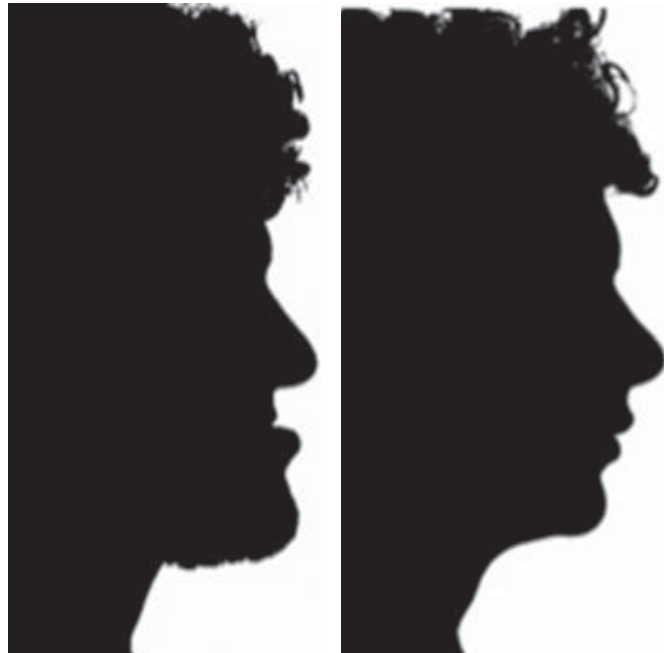

Figura 11: Comparativa perfil pre- y postoperatorio. Fuente: Hospital General «Dr. Manuel Gea González».
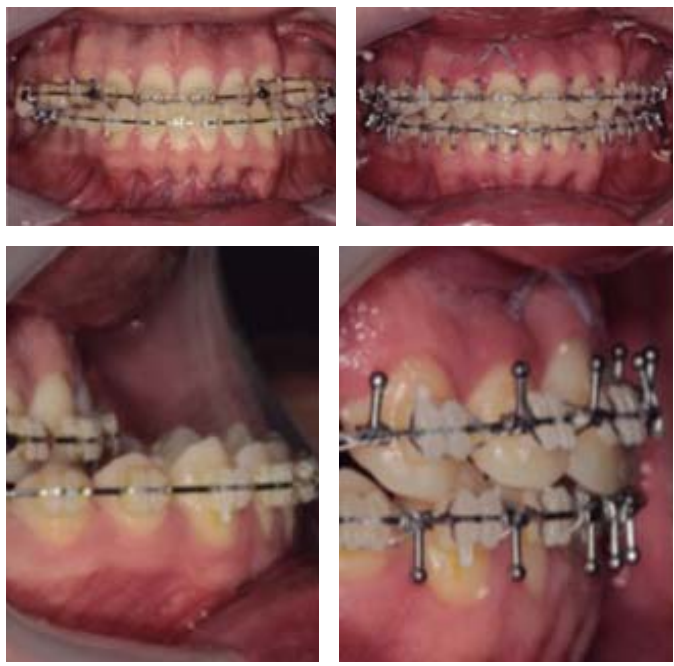

Figura 12: Fotografías comparativas mordida pre- y postoperatoria.

Fuente: Servicio de Ortodoncia. Hospital General «Dr. Manuel Gea González».

contracción supero-posterior produce una apariencia avejentada en forma prematura. ${ }^{10}$

\section{CONCLUSIÓN Y RECOMENDACIONES}

Cuando se realizan procedimientos combinados ambiciosos, como osteotomía LeFort I más osteotomía sagital mandibular en aquellos casos 


\section{Figura 13:}

Ortopantomografía comparativa pre- y

postoperatoria.

Fuente: Servicio de

Ortodoncia. Hospital

General «Dr. Manuel

Gea González».

Figura 14:

Radiografía

comparativa

anteroposterior

y lateral pre- y

postoperatoria.

Fuente: Servicio de

Ortodoncia. Hospital

General «Dr. Manuel

Gea González».
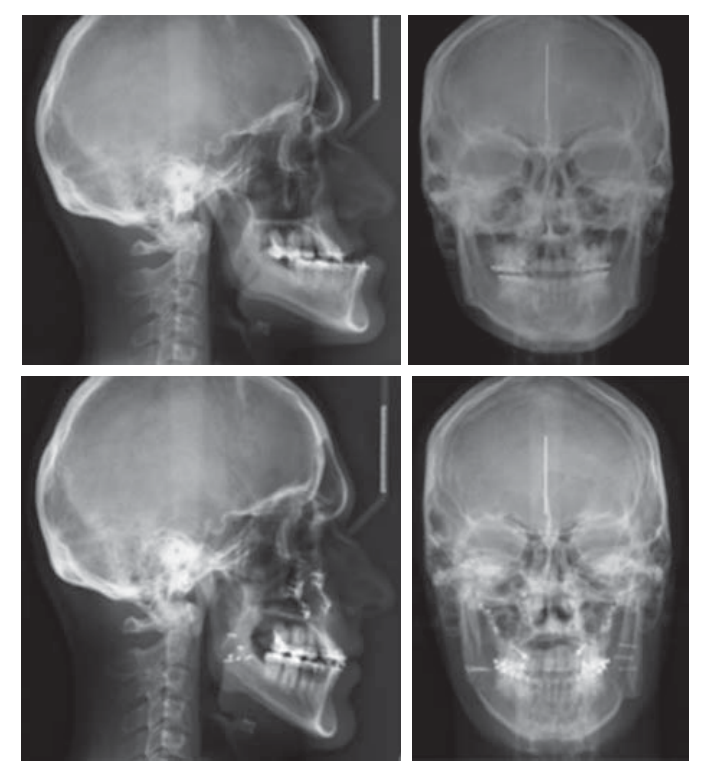

en los que están indicados, se puede lograr restablecer las adecuadas relaciones de los componentes maxilar y mandibular, además de lograr la corrección cefalométrica de la maloclusión, desproporción facial, o de la disfuncionalidad que no logran ser tratadas con ortodoncia o un procedimiento ortognático simple.

\section{REFERENCIAS}

1. Janis JE. Essentials of plastic surgery. UT Southwestern Medical Center. St. Louis Missouri 2007, pp. 259.

2. Nelligan PC. Plastic surgery. Department of Surgery, Division of Plastic Surgery. University of Washington, Seattle. 2013, pp. 664.

3. Ortiz Monasterio F, Molina F. Cirugía estética del esqueleto facial. México, Ed. Panamericana 2005, pp. 169-238.

4. Patel PK, et al. The Surgical Tools: The LeFort I, Bilateral sagittal split osteotomy of the mandible, and the osseous genioplasty. Clin Plast Surg 2007; 34: 447-475.

5. Da Silva de C.L. Consideraciones generales en el diagnóstico y tratamiento de las maloclusiones clase III. Revista Latinoamericana de Ortodoncia y Odontopediatría. Ortodoncia.ws edición electrónica julio 2005. Disponible en: www.ortodoncia.ws.

6. Talley Milan M, Katagiri Katagiri M, Pérez Tejada H. Casuística de maloclusiones clase I, clase II y clase III según Angle en el Departamento de Ortodoncia de la UNAM. Rev Odontol Mex 2007; 11 (4): 175-180.

7. Naran S, Steinbacher DM, Taylor JA. Current concepts in orthognathic surgery. Plast Reconstr Surg 2018; 141 (6): 925e-936e.

8. Potter JK. Basic oral surgery. Essentials of Plastic Surgery. UT Southwestern Medical Center. St. Louis Missouri 2007, pp. 265.

9. Grubb J, Evans C. Orthodontic management of dentofacial skeletal deformities. Clin Plast Surg 2007; 34 (3): 403-415.

10. Goldstein JA, Baker SB. Cleft and craniofacial orthognathic surgery. Plastic Surgery. Department of Surgery, Division of Plastic Surgery. University of Washington, Seattle 2013, pp. 665.

Conflicto de intereses: Los autores declaran no tener conflicto de intereses.

Correspondencia:

Dr. Jorge David Serrano Andrade

Calzada México Xochimilco Núm. 75, B202,

Pueblo San Lorenzo Huipulco, 14370,

Alcaldía Tlalpan, Ciudad de México, México.

E-mail: davichoserrano@gmail.com 